\title{
Análisis de concordancia inter-jueces para medir factores protectores en salud en universitarios
}

\section{Analysis of inter-judge agreement to measure protective factors in health in university students}

\section{Análise da concordância entre juízes para medir fatores de proteção em saúde em estudantes universitários}

\author{
Esperanza Cabrera-Díaz \\ Pedro León Reyes-Gaspar \\ Sergio Charry-Méndez
}

\section{Resumen}

Objetivo: Realizar el análisis de concordancia mediante el juicio de expertos de un instrumento de medición para detectar factores protectores de enfermedades no transmisibles en jóvenes universitarios. Materiales y método: Estudio psicométrico de validez de contenido, en el que participaron cuatro expertos que evaluaron cada ítem. Se determinó el nivel de concordancia entre jueces con el coeficiente Kappa de Fleiss. Resultados: Se observa fuerza de concordancia para el total de los ítems del instrumento como muy buena. En cuanto a los criterios para la significancia estadística fue de p: 0,000, los cuales se ajustaron a algunos ítems en redacción y contenido; luego se incorporaron algunos teniendo en cuenta las observaciones cualitativas de los expertos. Conclusión: El instrumento representa un avance en la medición del autocuidado de la salud en estudiantes Universitarios, el cual permitirá definir acciones para mejorar el estilo de vida en esta población.

Palabras claves: Promoción de la salud; estilo de vida; psicometría; enfermedad crónica; validación.

\begin{abstract}
Objective: To carry out the concordance analysis by means of the expert judgment of a measurement instrument to detect protective factors of non-communicable diseases in university students. Materials and method: Psychometric content validity study, in which four experts participated who evaluated each item, the level of agreement between judges with the Fleiss Kappa coefficient was determined. Results: strength of agreement is observed for the total items of the instrument, as very good, regarding the criteria for all the statistical significance was p: 0.000 , some items were adjusted in writing, content and some were incorporated taking into account Account for the qualitative observations of the experts. Conclusion: The instrument represents an advance in the measurement of health self-care in University students, which will allow defining actions to improve the lifestyle in this population.
\end{abstract}

Keywords: Health promotion; lifestyle; psychometry; chronic disease

\section{Resumo}

Objetivo: Realizar a análise de concordância por meio da perícia de um instrumento de medida para detectar fatores de proteção de doenças não transmissíveis em estudantes universi-
Autor de correspondencia*

${ }^{1}$ Psicóloga. Magister en Educación y Desarrollo Comunitario. Especialización y Doctorado en Bioética. Docente Tiempo Completo programa de Medicina Universidad Surcolombiana. Neiva, Colombia. Correo: cabreraesperanza@usco.edu.co (1) 0000-0001-8868$\underline{2832}$

${ }^{2}$ Medico Universidad del Central del Ecuador Quito. Especialización en Gerencia de Servicios de Salud y Epidemiologia. Doctorado en ciencias de la salud. Docente Tiempo Completo programa de Medicina Universidad Surcolombiana. Neiva, Colombia. Correo: reyesgaspar@usco.edu.co (D) 0000-00021597-409X

${ }^{3 *}$ Psicólogo Universidad Surcolombiana. Neiva, Colombia. Correo: sergio. charry@usco.edu.co (i) 0000-00020949-3791

Recibido: 4 junio 2021

Aprobado: 29 noviembre 2021

Para citar este artículo

Cabrera-Diaz E, Reyes-Gaspar PL, Charry-Méndez S. Análisis de concordancia inter-jueces para medir factores protectores en salud en universitarios. Rev. cienc. cuidad. 2022; 19(1):95-106 https://doi. org/10.22463/17949831.3055

(C) Universidad Francisco de Paula Santander. Este es un artículo bajo la licencia CC-BY-NC-ND 
tários. Materiais e método: Estudo psicométrico de validade de conteúdo, no qual participaram quatro experts que avaliaram cada item, foi determinado o nível de concordância entre juízes com o coeficiente Kappa de Fleiss. Resultados: a força de concordância é observada para todos os itens do instrumento, como muito boa, em relação aos critérios para todos a significância estatística foi p: 0,000 , alguns itens foram ajustados na escrita, conteúdo e alguns foram incorporados levando-se em conta a observações qualitativas dos especialistas. Conclusão: O instrumento representa um avanço na mensuração do autocuidado em saúde em universitários, o que permitirá definir ações para a melhoria do estilo de vida dessa população.

Palavras-chave: Promoção da saúde; estilo de vida; psicometria; doença crônica.

\section{Introducción}

El estilo de vida que adopta un individuo tiene repercusión en la salud tanto física como psíquica debido a que comprende hábitos que a la vez determinan la presencia de factores de riesgo, de factores protectores o ambos, de allí que sea importante tener en cuenta los comportamientos que rodean a cada individuo (1).

La adolescencia trae consigo modificaciones físicas, psicológicas y sociales las cuales transforman la vida de los jóvenes motivándolos a tener un papel activo en su propio autocuidado de la salud. En esta etapa se incorporan comportamientos riesgosos debido a la búsqueda de su independencia y de encajar en su ámbito social. Lo anterior se arraiga con el ingreso a la universidad y la adaptación a la vida universitaria. Se calcula que la carga total de morbilidad en los adultos está ligada a comportamientos que se inician durante la adolescencia (2). Por eso, la juventud representa una ocasión eficaz para el desarrollo de hábitos y estilos de vida saludables que perduraran por el resto de la vida (3). En este sentido es necesario trabajar las potencialidades vitales de los adolescentes, lo que les facilitará la comprensión de los riesgos y vulnerabilidades en la salud, ampliando el concepto de salud como un componente vital de la vida relacionado con lo cultural, lo social y lo económico (4).

Conviene subrayar que es necesario conocer los comportamientos de los adolescentes y ejercer influencia en ella; por tanto, es importante entender el entorno en que viven y cómo les afecta el desarrollo, la conducta y las relaciones sociales (5). Los centros de educación superior presentan ambientes propicios para el establecimiento de una adecuada promoción de la salud, ya que tienen una población adolescente en formación y con afianzamiento en estilos de vida. Al mismo tiempo dicha población acude a la universidad para prepararse como agente de cambio social a nivel profesional. Desde su actuar como mediadores, las universidades deben crear estrategias y programas de promoción de la salud adaptados a las necesidades locales y a sus posibilidades específicas. La participación activa en la promoción de la salud implica crear ambientes favorables que generen condiciones de vida gratificantes, agradables, seguras y estimulantes (6). Por otro lado, los jóvenes y la comunidad universitaria en general deben adquirir una nueva información que les permita elegir opciones saludables y disfrutar de un entorno que mejore su salud.

Es necesario recalcar que un factor puede en algunos casos ser de riesgo y/o protector por el desarrollo de nuevas actitudes o destrezas, según las circunstancias, las respuestas y el respaldo que encuentre en el proceso el individuo (7). Los factores protectores son características, rasgos, habilidades, competencias y medios que se pueden detectar, potencializar y desarrollar (sujeto, familia, grupo o comunidad) puesto que favorecen no solo el desarrollo humano sino también la reparación y sostenimiento de la salud; del mismo modo, tienen la capacidad de neutralizar las consecuencias de los efectos de los factores y conductas de riesgo (8). Los factores protectores pueden tener un amplio espectro, al facilitar una mayor probabilidad sobre la presencia de conductas protectoras que favorecen la no presencia de daños o riesgos; también pueden indicar ciertas conductas de riesgo $(8,9)$.

En lo que respecta a las Enfermedades No Transmisibles (ENT) estas se pueden prevenir y controlar, entre 


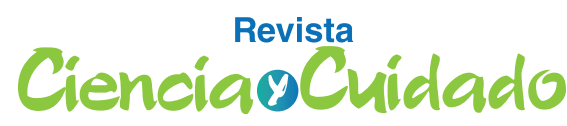

Scientific Journal of Nursing

otras acciones, a través de cambios en el estilo de vida; es decir, mediante factores protectores como mantener un peso adecuado, la práctica del ejercicio regular, la alimentación y dieta saludable y por último el no consumo de tabaco y alcohol (10). Estas prácticas están asociadas a una reducción de hasta el $80 \%$ en el riesgo de desarrollar las ENT más comunes (11-13).

Con respecto a las ENT como la enfermedad cardiovascular, la enfermedad respiratoria crónica, el cáncer y la diabetes (las cuatro ENT más comunes) representan en la actualidad un problema de salud pública (14), así como uno de los mayores riesgos de nuestro tiempo aumentando una creciente crisis de salud en las próximas décadas. Por su magnitud y letalidad las ENT son la principal causa de muerte en el mundo, no discriminan sexo y edad y tienen un mayor impacto en las personas con menor nivel de ingresos y educativo. En el mundo, 41 millones de personas cada año mueren por ENT; por tanto, corresponden al $70 \%$ de las defunciones anuales en todo el mundo (15). Hay que mencionar además, que este tipo de enfermedades predominaban en personas de la tercera edad; no obstante, actualmente 15 millones de personas mueren prematuramente entre los 30 y 69 años de edad (16). En Colombia más de 110 mil personas fallecen por ENT relacionadas con afecciones cardiovasculares, cáncer, diabetes y padecimientos pulmonares (17). El Instituto Nacional de Salud, en los resultados del séptimo análisis sobre la situación de salud evidenció que la principal causa de muerte en Colombia entre el 2005 y el 2013 fueron las enfermedades cardiovasculares y como conclusión final manifestaron que las ENT siguen siendo las principales causantes de muerte de los colombianos, encabezadas por los eventos cardiovasculares, seguidas del cáncer, la enfermedad pulmonar obstructiva crónica y la diabetes (18). Los factores de riesgo de las ENT tienen como causas principales: la alimentación poco saludable, la inactividad física y el consumo de tabaco y alcohol. Estos factores están asociados con comportamientos que se suelen instaurar durante la adolescencia y la juventud (19).

Por todo lo anterior es pertinente contar con un instrumento científicamente validado, con impacto social y contribución teórica que garantice la recolección de información y el análisis sobre factores protectores de ENT en jóvenes universitarios. Al respecto se tomó como modelo el Proyecto Institucional Usco Saludable, el cual promueve en la comunidad universitaria hábitos de vida saludables que conllevan al establec-
Análisis de concordancia inter-jueces para medir factores protectores en salud en universitarios imiento de una cultura de la salud; igualmente, desarrolla sus actividades desde un enfoque Sanológico. En tal sentido se vio la necesidad de construir y validar un instrumento para identificar los factores protectores de ENT en jóvenes universitarios, con el fin de describir su perfil actual de salud y así ofertar mejores servicios protectores, cuidado y promoción que contribuyan a la salud, al cambio social, al bienestar y a la calidad de vida de la comunidad universitaria en general.

Hay que especificar que en la búsqueda bibliográfica realizada no se encontró ni a nivel nacional ni internacional un instrumento como el que plantean los autores. $\mathrm{Y}$ en cuanto a los factores de riesgo los instrumentos de detección estaban aplicados a personas asintomáticas, siendo poco usuales en el primer nivel de atención. Por consiguiente, la información que se obtenga a través del instrumento propuesto permitirá identificar factores protectores que permitirán describir el perfil actual de salud y aumentar esfuerzos para promover factores protectores y mejorar la calidad de vida de los universitarios, proporcionándoles así un mayor control de su propia salud. Además facilitará aportar respuestas válidas que sirvan como alternativas de solución a las circunstancias adversas vividas en Colombia en este campo de la salud pública y las ENT.

\section{Objetivos}

\section{Objetivo general}

Determinar el análisis de concordancia mediante el juicio de expertos de un instrumento de medición para detectar factores protectores de enfermedades no transmisibles en jóvenes universitarios

\section{Objetivos específicos}

- Organizar el contenido del instrumento en dimensiones para adquirir la calificación de los jueces expertos

- Analizar y comparar los resultados de las calificaciones de los jueces expertos realizando la tabulación en el programa estadístico SPSS.

- Plantear modificaciones del instrumento mediante el análisis de resultados desarrollado por los jueces expertos, para hallar la validez de contenido 


\section{Materiales y métodos}

Esta investigación es tipo descriptivo, cuantitativo de corte trasversal, psicométrica de validez de contenido por medio de juicio de expertos. La población fue conformada por profesionales, expertos en el área de estilos de vida saludables, coordinación, seguimiento y evaluación de proyectos de prevención y manejo de enfermedades a nivel comunitario, promoción de la salud. Todos ellos con experiencia en docencia universitaria de más de 10 años e investigadores de nivel superior, con formación académica de Maestría y Doctorado. La muestra fue intencional, incluyó cuatro expertos de dif- erentes universidades públicas y privadas de Colombia, que aceptaron participar de forma voluntaria. Para la selección y participación de los jueces se tomaron los criterios establecidos por kjong y Wentworht (20), relacionados con la experiencia en la realización de juicios y toma de decisiones basada en evidencia o experticia (grados, investigaciones, publicaciones, posición, experiencia y premios entre otras), reputación en la comunidad, disponibilidad y motivación para participar e imparcialidad y cualidades inherentes como confianza en sí mismo y adaptabilidad. También plantean que los expertos pueden estar relacionados por educación similar, entrenamiento, experiencia o número de publicaciones.

Tabla 1. Criterios jueces expertos

\begin{tabular}{ccccc}
\hline Juez & Nivel educativo & Grado de Investigador & Índice H & Experiencia \\
\hline 1 & Ph.D & Senior & H 10 & 15 \\
2 & Ph.D & Junior & H 10 & 11 \\
3 & Ph.D & Junior & H 5 & 13 \\
4 & Magister & Asociado & H 2 & 11 \\
\hline
\end{tabular}

Fuente: Elaboración propia

El instrumento para detectar los factores protectores de enfermedades no transmisibles en jóvenes universitarios, se desarrolló como parte de este estudio y tiene como función encontrar un grupo de factores que pueden ser protectores para la salud de los jóvenes universitarios. Está conformado por 90 ítems que hacen referencia a los cuatro factores protectores más influyentes en la prevención y promoción de las ENT: Actividad física. hábitos alimenticios, consumo de tabaco y consumo de alcohol. De igual manera se efectuó la revisión de la literatura y otros instrumentos sobre factores de protección y estilos de vida saludable. El proceso incluyó la extracción de ítems claves procedentes de instrumentos como: cuestionario de prácticas y creencias sobre estilos de vida, cuestionario de estilo de vida, cuestionario CAGE, cuestionario sobre factores de protección ante el consumo de tabaco y alcohol. En consonancia con lo expuesto anteriormente se seleccionaron estos instrumentos ya que poseen propiedades psicométricas confiables. Igualmente se construyeron otros ítems con base en la teoría encontrada sobre las áreas de interés. Se responde mediante una escala tipo Likert del 1 a 3 , en donde 1 es nunca y 3 es casi siempre.

Para establecer los indicadores se tuvieron en cuenta los cuatro factores de protección: alimentación saluda- ble, actividad física, consumo de alcohol y tabaco; así como para las cuatro ENT más comunes: enfermedad cardiovascular, cáncer, enfermedades respiratorias y diabetes. La redacción de los ítems obedece a los factores protectores y se hicieron con base en la edad y la escolaridad de los jóvenes. El instrumento no requiere de previa capacitación ni contar con características específicas (como el tiempo de respuesta) para ser aplicado, salvo que el contexto de estudio sea la universidad, en función de que es un instrumento para identificar la protección de los jóvenes universitarios ante las ENT.

Posterior a la selección y construcción de los ítems se sometió el instrumento a la evaluación por juicio de expertos. Como referente metodológico para el proceso de validez se tomó la propuesta de Escobar y $\mathrm{Cu}$ ervo (21), quienes diseñaron una plantilla con cuatro criterios: suficiencia, claridad, coherencia y relevancia. Por otra parte, al evaluar el instrumento se tuvieron en cuenta estos criterios, los cuales midieron la concordancia entre los cuatro jueces evaluadores expertos. Cada experto dio su juicio para cada ítem, a partir de las opciones de respuesta, con una evaluación según la Escala tipo Likert, que constaba de un intervalo de 1 a 4 , donde el valor 1 no cumple y 4 es un nivel alto de aceptación del ítem (Tabla 2). 
Scientific Journal of Nursing

Tabla 2. Criterios de evaluación de la concordancia inter-jueces de un instrumento

\begin{tabular}{|c|c|c|}
\hline Dimensión & Conceptos & Categorías \\
\hline Suficiencia & $\begin{array}{l}\text { Los ítems que pertenecen a una mis- } \\
\text { ma dimensión bastan para obtener la } \\
\text { medición de ésta. }\end{array}$ & $\begin{array}{l}\text { No cumple } \\
\text { Nivel bajo } \\
\text { Nivel moderado } \\
\text { Nivel alto }\end{array}$ \\
\hline Claridad & $\begin{array}{l}\text { El ítem se comprende fácilmente, es } \\
\text { decir, su sintáctica y semántica son } \\
\text { adecuadas. }\end{array}$ & $\begin{array}{l}\text { No cumple } \\
\text { Nivel bajo } \\
\text { Nivel moderado } \\
\text { Nivel alto }\end{array}$ \\
\hline Coherencia & $\begin{array}{l}\text { El ítem tiene relación lógica con la } \\
\text { dimensión o indicador que está midi- } \\
\text { endo. }\end{array}$ & $\begin{array}{l}\text { No cumple } \\
\text { Nivel bajo } \\
\text { Nivel moderado } \\
\text { Nivel alto }\end{array}$ \\
\hline Relevancia & $\begin{array}{l}\text { El ítem es esencial o importante, es } \\
\text { decir debe ser incluido. }\end{array}$ & $\begin{array}{l}\text { No cumple } \\
\text { Nivel bajo } \\
\text { Nivel moderado } \\
\text { Nivel alto }\end{array}$ \\
\hline
\end{tabular}

Fuente: Galicia-Alarcón, Balderrama-Trapaga, Edel-Navarro adaptado de Escobar y Cuervo (2017).

Para contactar a los jueces se les envió una carta de invitación vía correo electrónico explicando los objetivos de la prueba, el propósito del instrumento y demás descripciones para contextualizarlos; igualmente, se adjuntó el instrumento y la plantilla para su evaluación según los parámetros establecidos. Se les concedió un plazo máximo de 30 días a partir de la confirmación de participación de cada juez, para realizar la evaluación y entregar de forma digital sus apreciaciones.

Por otra parte, en función de los hallazgos de la primera revisión por los cuatro jueces expertos, se realizaron ajustes al instrumento tales como: eliminar ítems que eran irrelevantes, incorporar ítems que eran necesarios y modificar aquellos que lo requerían según los expertos. Antes de la primera revisión, el instrumento estaba compuesto por 78 ítems y posteriormente quedó con 90 ítems.

Una vez efectuados los cambios, el instrumento es nuevamente enviado a los jueces para una segunda evaluación. Por último, se ejecutó el análisis estadístico para establecer el grado de concordancia entre los jueces. Para esto se implementó el estadístico Kappa de Fleiss.

Para el análisis estadístico se trasladó la información al programa SPSS, IBM Versión 22, aplicando la prueba no paramétrica de Kruskal- Wa $\neg l$ lis para muestras independientes, con un nivel de significación asintótica de $p=0,05$, a fin de identificar si la distribución de las dimensiones: suficiencia, claridad, coherencia y relevancia, era la misma entre la categoría jueces (4). Para el análisis de concordancia interjueces se empleó Kappa de Fleiss con la fórmula propuesta por Cohen, pero adaptada para más de dos codificadores. El coeficiente Kappa de Fleiss (22), añade el cálculo del sesgo del codificador (precisión-error) y el cálculo de la concordancia (calibración). El nivel de significancia establecido fue de $\mathrm{p}=0,05$.

Cabe señalar que para la interpretación del índice Kappa de Fleiss se utilizaron las categorías propuestas por Altman (23), las cuales permitieron interpretar los coeficientes alcanzados. El autor presenta valores que oscilan de 0 a 1 , en donde 0 indica mayor desacuerdo entre los jueces y 1 mayor grado de acuerdo. La clasificación muestra que los coeficientes de kappa pueden ser pobres $(0$ a 0.20$)$, débiles $(0,21$ a 0,40$)$, moderados $(0.41$ a 0.60$)$, buenos $(0.61$ a 0.80$)$ y muy buenos $(0.81$ a 1.00).

Hay que señalar que en el desarrollo de esta investigación se tuvo en cuenta la Resolución 8430 de Octubre de 1993, la cual establece normas científicas, técnicas y administrativas para la investigación en salud y aspectos éticos en la investigación con seres humanos. Por sus características ésta es una investigación con riesgo mínimo, dado que la recolección de datos se hará a través de procedimientos sencillos que no ponen en riesgo la salud ni la integridad de los sujetos de estudio. 


\section{Resultados}

Se aplicó la prueba no paramétrica de Kruskal- Wallis para muestras independientes, con un nivel de significación asintótica de $\mathrm{p}=0,05$ para ver si la distribución de las dimensiones como suficiencia, claridad, coherencia y relevancia, que miden la concordancia o niv- el de acuerdo, era la misma entre la categoría jueces $(n=4)$. Como no se encontraron diferencias significativas para cada dimensión (tabla 3), se puede inferir que existe acuerdo entre los jueces; de ahí, que se acepte la hipótesis nula de que la distribución para cada una de las dimensiones sobre suficiencia, claridad, coherencia y relevancia es la misma para la categoría de los jueces

Tabla 3. Contrastes de hipótesis, Prueba Kruskal-Wallis para muestras independientes según la categoría Juez (Cuatro jueces).

\begin{tabular}{|c|c|c|c|}
\hline Hipótesis Nula & Prueba & Sig. & Decisión \\
\hline $\begin{array}{l}\text { Distribución de Suficiencia es } \\
\text { la misma entre los jueces. }\end{array}$ & $\begin{array}{l}\text { Kruskas-wallis para } \\
\text { muestra independientes }\end{array}$ & ,409 & $\begin{array}{l}\text { Conserve la hipótesis } \\
\text { nula }\end{array}$ \\
\hline $\begin{array}{l}\text { Distribución Coherencia es la } \\
\text { misma entre los jueces. }\end{array}$ & $\begin{array}{l}\text { Kruskas-wallis para } \\
\text { muestra independientes }\end{array}$ &, 082 & $\begin{array}{c}\text { Conserve la hipótesis } \\
\text { nula }\end{array}$ \\
\hline $\begin{array}{l}\text { Distribución Coherencia es la } \\
\text { misma entre los jueces }\end{array}$ & $\begin{array}{l}\text { Kruskas-wallis para } \\
\text { muestra independientes }\end{array}$ & ,392 & $\begin{array}{c}\text { Conserve la hipótesis } \\
\text { nula }\end{array}$ \\
\hline $\begin{array}{l}\text { Distribución Relevancia es la } \\
\text { misma entre los jueces. }\end{array}$ & $\begin{array}{l}\text { Kruskas-wallis para } \\
\text { muestra independientes }\end{array}$ & ,392 & $\begin{array}{c}\text { Conserve la hipótesis } \\
\text { nula }\end{array}$ \\
\hline
\end{tabular}

Fuente: visor de resultado Spss 22

Una vez identificada la distribución de las categorías entre los jueces, se realizó la primera evaluación mediante el coeficiente de kappa de Fleiss permitiendo evidenciar: primero, que el nivel de concordancia establecido por los jueces expertos para los ítems en las cuatro categorías para cada una de las dimensiones del cuestionario estimó la fuerza de concordancia como no significativa, interpretada como pobre, como se describe en la tabla 4. Segundo, se ajustó la estructura del instrumento. De los 78 ítems iniciales se eliminaron 2 y se incorporaron 14 .

Tabla 4. Resultados de la primera medición de Kappa de Fleiss para las dimensiones suficiencia, claridad, coherencia y relevancia.

\begin{tabular}{cccc}
\hline Dimensión & $\begin{array}{c}\text { Categoría Kappa de } \\
\text { Fleiss }\end{array}$ & Concordancia interjueces & P \\
\hline Suficiencia & $\mathrm{K}=-0,074$ & 0.20 Pobre & 0,394 \\
Claridad & $\mathrm{K}=0,08$ & 0.20 Pobre & 0,342 \\
Coherencia & $\mathrm{K}=-0,02$ & 0.20 Pobre & 0,872 \\
Relevancia & $\mathrm{K}=0,02$ & 0.20 Pobre & 0,872 \\
\hline
\end{tabular}

Fuente: visor de resultado Spss 22. 


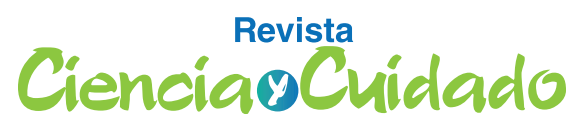

Scientific Journal of Nursing

En cuanto a la significancia estadística de las categorías, se presentó un comportamiento del 95\% tomando como umbral de significancia el valor $p=0,05$, siendo notables las categorías de coherencia y relevancia con $p=0,872$ (tabla 4) lo que demostró una mayor diferencia en las apreciaciones de los jueces expertos. En definitiva, es necesario mejorar estos indicadores teniendo en cuenta las observaciones presentadas por los jueces expertos.

De acuerdo con las observaciones de los jueces expertos se realizaron las siguientes modificaciones al cuestionario: se incorporaron ítems relacionados a las actitudes positivas y ambiente familiar haciendo que los factores de riesgo fueran incompatibles, puesto que la ausencia de estos indicadores afectó la suficiencia del cuestionario en la primera evaluación. Los ítems referentes a las actitudes positivas fueron los siguientes: Durante el año participa de eventos locales que promocionan la realización de actividades fisicas (torneos, festivales, concursos, maratones, carreras) o de dias temáticos alusivos a la actividad fisica; posee en su celular o dispositivos móviles aplicaciones que le ayuden a realizar y monitorear su actividad física (como pasómetro, medidores de frecuencia cardiaca, entre otros); utiliza los espacios entre clases para moverse (hacer alguna actividad fisica). Durante las clases los profesores realizan activaciones; participa de la oferta institucional para hacer algún tipo de actividad fisica, deportiva o lúdica; considera que el costo de los alimentos saludables es alto y tiene tiempo para preparar sus propios alimentos. Los ítems incorporados en relación al ambiente familiar fueron los siguientes: Durante el año su familia participa de eventos locales que promocionan la realización de actividades físicas (torneos, festivales, concursos, maratones, carreras) o de dias temáticos alusivos a la actividad fisica. Su familia hace uso de parques, ciclo-rutas, escenarios deportivos y/o senderos ecológicos; programan en familia tiempos comunes para disfrutar de actividades como caminar, montar bicicleta, patinar, pasear la mascota, elevar cometas, hacer un picnic. Practican juegos de actividad en familia como alternativa a los juegos tradicionales de mesa; en su casa hay algún espacio concertado para realizar algún tipo de actividad física según las preferencias de los miembros de la familia; algún miembro de su familia prepara sus alimentos $d u$ rante la semana; cuando su familia cocina, lo hace sin
Análisis de concordancia inter-jueces para medir factores protectores en salud en universitarios adicionar sal (cocina sin sal).

De igual manera se eliminaron dos ítems: "En la universidad se realizan campañas sobre el abuso del alcohol y sus consecuencias. La universidad ofrece espacios libres 100\% de humo", los cuales estaban argumentados desde un contexto social que puede tener influencia en la adquisición de una conducta protectora; sin embargo, para los jueces la decisión final siempre está ligado a la toma de decisiones personales. Por esta razón las características individuales presentan un valor sobresaliente en la fundación y mantenimiento de conductas protectoras, ya que cada persona tiene un significado y valor a los dominios sociales, ambientales y familiares; por tanto, estos aspectos afectaron la claridad y suficiencia de los ítems en la primera evaluación.

Las contribuciones de los jueces están orientadas al mejoramiento de los ítems, pero también están dirigidas sobre aspectos globales como los redactados en forma negativa que debieron ser restructurados, puesto que pueden involucrar una doble negación; razón por la cual proponen direccionarlo de forma positiva. "No me parece conveniente estar cerca de personas que fuman" se puede cambiar por la afirmación "Evita estar cerca de personas que fuman" (juez 3). En consecuencia, 15 ítems fueron corregidos y redactados en forma positiva. Estos aspectos afectaron la claridad y relevancia en la primera evaluación. De este modo, junto a las opiniones de los jueces expertos que se indican en cada ítem, se revisa y reestructura el contenido del instrumento hasta obtener ítems coherentes, claros, suficientes y relevantes. Finalizado este proceso el instrumento se remite nuevamente a los expertos para efectuar la segunda evaluación.

Una vez sometido el cuestionario a la segunda evaluación por los expertos en cuanto al grado de concordancia se evidenció un mejoramiento sustancial en cada dimensión y categoría evaluada para cada ítem. El Kappa de Fleiss indica que los niveles son significativos $(\mathrm{p}=$ $0,000)$, pasando de la categoría pobre a Muy Buena $(\mathrm{k}=0,81-1,00)$ (tabla 5). Por lo anterior se considera que el instrumento es válido, ya que los cuatro jueces expertos están de acuerdo en que los ítems presentan niveles muy buenos de suficiencia, claridad, coherencia y relevancia. 
Tabla 5. Resultados segunda evaluación jueces expertos

\begin{tabular}{cccl}
\hline Dimensión & Kappa de Fleiss & P & Categorías \\
\hline Suficiencia & $\mathrm{K}=0,85$ & 0,001 & $0,81-1,00$ Muy Buena \\
Claridad & $\mathrm{K}=0,88$ & 0,000 & $0,81-1,00$ Muy Buena \\
Coherencia & $\mathrm{K}=0,82$ & 0,001 & $0,81-1,00$ Muy Buena \\
Relevancia & $\mathrm{K}=1,00$ & 0,000 & $0,81-1,00$ Muy Buena \\
\hline
\end{tabular}

Fuente: visor de resultado Spss 22.

\section{Discusión}

La intención de este artículo ha sido la validación de contenido de un instrumento para detectar un grupo de factores que pueden ser protectores para la salud de los jóvenes universitarios, mediante la técnica de juicio de expertos, pues además de ser un método tratado para validaciones de contenido, evidencia seguridad en la técnica de validación $(24,25)$. Motivo por el cual se construyó un cuestionario de 90 afirmaciones compuestas por los cuatro factores protectores más influyentes en la prevención y promoción de las ENT más comunes: Actividad física, hábitos alimenticios, consumo de tabaco y consumo de alcohol.

Al respecto se realizó una validación de contenido donde participaron cuatro especialistas sobre estilos de vida saludable y por medio del coeficiente del Kappa de Fleiss mostró un resultado del grado de concordancia entre los jueces de $\mathrm{K}=0,81-1,00$, lo que permite concluir que los ítems del cuestionario y sus respectivas escalas establecen un nivel muy bueno de suficiencia, , coherencia y relevancia; además, se concluye que el cuestionario es idóneo para determinar el fenómeno estudiado. Aunque este proceso es importante, sin embargo, es indispensable que el cuestionario se someta a otros procesos de validez y fiabilidad. Durante el proceso de validación se pudo observar que la mayoría de los ítems muestran que existe un alto nivel de concordancia entre los jueces evaluadores.

Vale la pena subrayar, que en el proceso de evaluación de juicio por parte de expertos fue necesario reestructurar el instrumento y someterlo nuevamente a evaluación ya que algunos ítems tenían un bajo nivel de concordancia. Por otra parte, el nivel de experiencia que presenta cada uno de los jueces en la temática desarrollada es un punto a favor en la investigación, ya que cada uno contaba con la formación y experiencia suficiente para determinar cada aspecto del cuestionario, evitándose sesgos determinados por la subjetividad de las respuestas de los evaluadores, lo que dio validez a los resultados obtenidos.

Hay que precisar que un problema esencial en el proceso de validación de contenido está relacionado con el número indicado de expertos que deberían participar en el proceso, el cual no presenta un consenso absoluto entre diferentes autores, pues algunos señalan que la cantidad idónea debería ser entre 4 y 10 expertos $(26,27)$; sin embargo, otros consideran que la cantidad debería ser 3 como mínimo (28), dependiendo de los objetivos y el tipo de investigación a realizar; en cambio otros argumentan que el número de expertos debe oscilar entre 2 y hasta 20 (29) para el desarrollo de la validación de contenido. Se debe agregar que para este estudio se focalizaron 7 expertos, de los cuales 4 aceptaron la invitación a participar de la validación, es decir, que este número concuerda con la cantidad mínima que se necesita para efectuar la validez de contenido de un instrumento.

Los resultados que se obtuvieron en la presente investigación coinciden con los de Bernal, Salamanca, Pérez y Quemba (25), quienes validaron el contenido de un instrumento diseñado para estudiantes universitarios utilizando el coeficiente kappa de Fleiss como procesamiento analítico, para determinar el valor estadístico de la validez. En esta investigación participaron 9 expertos, quienes evaluaron cada ítem con base en las categorías de: suficiencia, claridad, coherencia y relevancia. Globalmente obtuvieron resultados casi perfectos $(0,81-1,00)$ en la concordancia interjueces para las características; mientras que la pertinencia fue la más alta $(0,8443)$ y en la significación estadística, es la suficiencia con $(p=0,0268)$.

De igual manera se encontró asociación con el trabajo realizado por Cabrera, Astaiza y Charry (13) en don- 


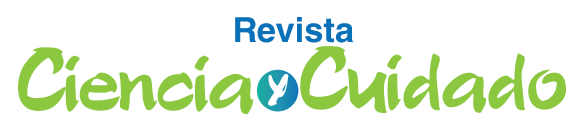

Scientific Journal of Nursing

de se efectuó un instrumento denominado Factores de riesgo para Enfermedades crónicas no trasmisibles en estudiantes universitarios, cuya validez de contenido se consiguió mediante el coeficiente kappa de Fleiss. Para efectuar este estudio los autores citados consultaron a 5 expertos obteniendo como resultados la reestructuración y eliminación de algunos de los ítems del instrumento mediante un $\mathrm{p}=000$ significativo en cada categoría. Sin embargo, el instrumento difiere de la visión positiva que se emplea en el actual cuestionario, ya que retoma los factores de riesgo estableciendo una visión dramática y un modelo de déficit en esta etapa de la vida.

Se sabe que la universidad es un espacio para la adquisición y modificación de estilos de vida, los cuales pueden influenciar a toda la comunidad, a través una serie de variables como: la edad, la socialización, la migración y la disfuncionalidad familiar, entre otras. Estos factores hacen que de una u otra manera esta población se más vulnerable adoptando hábitos pocos saludables y acentuando comportamientos en la vida estudiantil relacionados con: la mala alimentación, el sedentarismo, el estrés, la ansiedad, la depresión, el consumo excesivo de bebidas y sustancias psicoactivas $(13,30-33)$.

Por consiguiente, establecer un adecuado esquema del autocuidado de la salud, depende de la responsabilidad por parte de cada individuo sobre su bienestar. La toma de decisiones juega un papel importante en la vida de las personas, puesto que cada decisión afecta de manera indirecta o directa el estado de la salud. Por esta razón es importante que en el proceso de construcción del individuo en su ser y actuar, se pueda contar con las herramientas primordiales que le faciliten mantener un estado de salud óptimo y un desarrollo apropiado sobre su autocuidado.

Definitivamente, recolectar datos sobre las ENT desde la promoción permitiría contar con un registro sobre una temática que no se ha explorado a profundidad, permitiendo el surgimiento de nuevas propuestas frente a la salud de la población joven. Desde lo teórico el cuestionario es un instrumento útil que se puede aplicar para la promoción de la salud; de ahí, que su función sea transversal al afectar a cada nivel de atención integral. En consecuencia, se espera contar con un cuestionario con enfoque protector que amplíe la información que se conoce sobre los estilos de vida de los jóvenes, las
Análisis de concordancia inter-jueces para medir factores protectores en salud en universitarios

tendencias en hábitos saludables y sus recursos frente a la promoción de la salud, así como sus progresos. Por todo lo dicho anteriormente, lo técnico y metodológico permitirían establecer estrategias y realizar propuestas de mejora específicas para una población que tiene todo el potencial para incrementar el control sobre su salud y mejorarla.

Desde lo práctico, igualmente los resultados que arroje el instrumento servirán para orientar y definir las acciones que se deban tomar para la población, específicamente la universitaria; es decir, que servirá para llevar a cabo acciones coherentes frente a necesidades puntuales relacionadas con los estilos de vida, especialmente por parte de los profesionales implicados directamente en la promoción de la salud. Sumado a lo anterior, también, se podrá evaluar si las acciones tomadas con anterioridad realmente están o no cumpliendo su papel como barrera protectora en los jóvenes, al permitirles un aprendizaje consciente sobre su propia salud y la salud colectiva; por tanto, deben optar por lo saludable, que es una decisión fácil de tomar.

Conviene subrayar que el instrumento evaluado representa un avance en la medición de los factores de protección asociados con los estilos de vida de los estudiantes, al cumplir el objetivo principal como era el de validar el contenido del cuestionario. Por consiguiente podría ser examinado y aplicado en diferentes comunidades académicas a fin de detectar factores protectores de enfermedades no transmisibles en jóvenes universitarios; de igual forma se pueden aplicar nuevos ensayos psicométricos que ayuden a elevar su nivel de validez, para establecer acciones de aprendizaje sobre el autocuidado de la salud.

\section{Conclusiones}

- El instrumento para medir factores protectores sobre ENT en universitarios dispone de niveles muy buenos de análisis de concordancia con los inter-jueces, indicando que sirve para determinar comportamientos desde el enfoque protector y ampliar la información sobre estilos de vida en jóvenes universitarios.

- La técnica de juicio de expertos es una buena herramienta que garantiza seguridad en la validación, elaboración y evaluación de instrumentos de medición. 
- Utilizar el instrumento en otras investigaciones permitirá definir las acciones para promover un estilo de vida saludable, basado en un enfoque protector que abarque todas las dimensiones de la vida de los estudiantes universitarios.

- Para los autores el instrumento representa un avance en la caracterización de los factores protectores en población preadolescente y adolescencia inicial.

\section{Conflicto de intereses}

Los autores declaran no tener ningún conflicto de interés

\section{Referencias Bibliográficas}

1. Barbosa-Granados SH, Urrea-Cuellar AM. Influencia del deporte y la actividad física en el estado de salud físico y mental: una revisión bibliográfica. Revista Katharsis. [Internet]. 2018 [Consultado 26 de mayo del 2021]; 25:141-159. Disponible en: http://revistas.iue.edu.co/index.php/katharsis

2. Organización Mundial de la Salud. Los jóvenes y los riesgos sanitarios. [Internet] 2018 [consulta?do 20 de mayo del 2021] Disponible en: https://www.who.int/es/news-room/fact-sheets/detail/adolesᄀcents-health-risks-and-solutions

3. Angelucci LT, Cañoto Y, Hernández MJ. Influencia del estilo de vida, el sexo, la edad y el IMC sobre la salud física y psicológica en jóvenes universitarios. Avances en Psicología Latinoamericana. [Internet]. 2017 [Consultado 26 de mayo del 2021]; 35(3):531- 546. Disponible en: http://dx.doi.org/10.12804/10.12804/revistas. urosario.edu.co/apl/a.4454

4. Cabrera-Díaz E, Charry-Méndez S. Promoción del autocuidado de la salud en estudiantes universitarios. Proyección social [Internet] 2020 [consultando 29 de mayo del 2021]; 4(1):54-62. Disponible en: https:// jour-nalusco.edu.co/index.php/rps/article/view/3028/4089

5. Borrás-Santiesteban T, Reynaldo-Borrás A, López-Domínguez M. Adolescentes: razones para su atención. ccm [Internet]. 2017 [Consultado 26 de mayo del 2021]; 21(3):858-875. Disponible en: http://scielo.sld.cu/ scielo.php?script $=$ sci_arttext\&pid $=\mathrm{S} 1560-43812017000300020 \& \operatorname{lng}=\mathrm{es}$

6. Cabrera-Díaz E, Charry-Méndez S. Promoción del autocuidado de la salud en estudiantes universitarios. Revista proyección social [Internet]. 2021 [consultado el 26 de mayo del 2021]; 4(1):54-62. Disponible en: https://journalusco.edu.co/index.php/rps/article/view/3028

7. Bonilla-Ruiz SM, Marcilla-Hernández Y. Factores protectores que favorecen la realización de proyecto de vida en adolescentes en riesgo psicosocial de la Institución Juventud con una Misión, 2016. [Tesis en internet]. Corporación Universitaria Lasallista. Caldas, Antioquia. Colombia. [Consultado el 27 de mayo del 2021]. Disponible en: http://repository.lasallista.edu.co/dspace/handle/10567/1698

8. Muñoz C, Sandstede M, Klimenko O. Factores de riesgo y de protección para el consumo de drogas en los estudiantes del Instituto de Filosofía de la Universidad de Antioquia, 2016. Revista Psicoespacios. [Internet] 2017 [Consultado el 25 de mayo del 2021];11(18):149-170. Disponible en: http://revistas.iue.edu.co/index. php/Psicoespacios

9. Fernandez-Castillo E, Molerio-Perez O, Herrera-Jiménez LF, Grau R. Validez y confiabilidad del cuestionario para evaluar factores protectores de la salud mental en estudiantes universitarios. Actualidades en psicología [Internet]. 2017 [Consultado el 27 de mayo del 2021] 31(122):103-117. Disponible en: https://revistas.ucr. ac.cr/index.php/actualidades/article/view/24584

10. Ministerio de Salud y Protección Social. Prevención de enfermedades no transmisibles. [Internet]. 2019 [Consultado el 24 de mayo del 2021]. Disponible en: https://www.minsalud.gov.co/salud/Paginas/Enfermedades-no-transmisibles.aspx

11. Pulido-Medina C, Reyes-Suarez N, León-Aristizabal A, Cárdenas-Casallas J, Rivera-Damián S, RodríguezSáenz A. Factores de Riesgo de enfermedades crónicas no trasmisibles en estudiantes de ciencias básicas de la Escuela Medicina de la Universidad Pedagógica. Salud Historia y Sanidad. [Internet]. 2015[Consultado el 
Scientific Journal of Nursing

25 de mayo del 2021];10(1):15-21. Disponible en: http://agenf.org/ojs/index.php/shs/article/view/2

12. Organización Panamericana de la Salud. Plan de acción para la prevención y el control de las enfermedades no transmisibles en las Américas 2013-2019. [Internet]. 2016 [Consultado el 28 de mayo del 2021]. Disponible en: https:/www.paho.org/hq/dmdocuments/2014/NCD-SP-low.pdf

13. Cabrera-Diaz E, Astaiza-Arias GM, Charry-Méndez SA. Factores de riesgo para enfermedades no trasmisibles en universitarios: validación de contenido de un cuestionario. Tempus Psicológico. [Internet]. 2021 [Consultado el 27 de mayo del 2021]; 4(1):67-83. Disponible en: https://doi.org/10.30554/tempuspsi.4.1.33378.202

14. Serra-Valdés MA, Serra-Ruiz M, Viera-García M. Las enfermedades Crónicas no trasmisibles: magnitud actual y tendencias futuras. Revista Finlay. [Internet]. 2018 [Consultado el 27 de mayo del 2021]; 8(2):140-148. Disponible en: http://www.revfinlay.sld.cu/index.php/finlay/article/view/561

15. Organización Mundial de la Salud. Prevención de Enfermedades no Trasmisibles en Adolescentes y Jóvenes. [Internet]. 2019[Consultado el 26 de mayo del 2021] Disponible en: https:/www.paho.org/hq/index. php?option $=$ com content $\&$ view $=$ article $\& i d=6680: 2012$-preventing-non-communicable-diseases-adolescents-young-adults\&Itemid=135\&lang=es

16. Organización Mundial de la Salud. Prevención y control de las enfermedades no trasmisibles: Datos y cifras. [Internet]. 2021[Consultado el 27 de mayo del 2021]. Disponible en: https:/www.who.int/es/news-room/factsheets/detail/noncommunicable-diseases

17. Organización Mundial de la Salud. Enfermedades no trasmisibles diseño de servicios de salud acorde con esquemas de protección social. [Internet]. 2017 [Consultado el 25 de mayo del 2021]. Disponible en: https:// www.paho.org/chi/index.php?option $=$ com content\&view $=$ article\&id=128:enfermedades-no-transmisi$\underline{\text { bles\&Itemid }=213}$

18. Ministerio de Salud y Protección Social. Análisis de situación de Salud (ASIS) Colombia 2016. Dirección de Epidemiologia y demografía. Bogotá, noviembre. [Internet]. 2016 [Consultado el 26 de mayo del 2021]. Disponible en: https://www.minsalud.gov.co/sites/rid/Lists/BibliotecaDigital/RIDE/VS/ED/PSP/asis-colombia-2016.pdf

19. Organización Mundial de la Salud. Salud del Adolescente y Joven adulto. [Internet]. 2021 [Consultado el 26 de mayo del 2021]. Disponible en: https:/www.who.int/es/news-room/fact-sheets/detail/adolescents-health-risks-and-solutions

20. Dorantes-Nova JA, Hernández-Mosqueda JS, Tobón-Tobón S. Juicio de expertos para la validación de un instrumento de medición del síndrome de Burnout en la docencia. Revista Ra Ximhai. [Internet]. 2016 [Consultado el 30 de mayo del 2021];12(6):327-346. disponible en: https://www.redalyc.org/pdf/461/46148194023.pdf

21. Galicia-Alarcón LA, Balderrama-Trapaga JA, Edel-Navarro R. Validez de contenido por juicio de expertos: propuesta de una herramienta virtual. Revista Apert. (Guadalaj., Jal.), Guadalajara. [Internet]. 2017 [Consultado el 30 de mayo del 2021]; 9(2):42-53. Disponible en: http://www.scielo.org.mx/scielo.php?script=Sci arttext\&pid=S1665-61802017000300042\&lng=es\&nrm=iso

22. Moya-Mata I, Martin-Ruiz J, Ruiz-Sanchis L, Ros-Ros C. Diseño Fiabilidad y validez de una herramienta para el análisis de las imágenes de los libros de texto de educación física. Revista Retos. [Internet]. 2018 [Consultado el 29 de mayo del 2021]; 34:240-246. Disponible en: https://recyt.fecyt.es/index.php/retos/article/view/82565

23. Manterola C, Grande L, Otzen T, García N, Salazar P, Quiroz G. Confiabilidad, precisión o reproducibilidad de las mediciones. Métodos de valoración, utilidad y aplicaciones en la práctica clínica. Revista Chilena Infectol. [Internet]. 2018 [Consultado el 30 de mayo del 2021]; 35(6):680-688. Disponible en: https://scielo. conicyt.cl/scielo.php?script $=$ sci serial\&pid=0716-1018

24. Galicia-Alarcon L, Balderrama-Trápaga JA, Edel-Navarro R. Validez de contenido por juicio de expertos: propuesta de una herramienta virtual. Revista Apertura. [Internet]. 2017 [Consultado el 28 de mayo del 2021]; 17(27):42-53. Disponible en: http://www.scielo.org.mx/pdf/apertura/v9n2/2007-1094-apertura-9-02-00042. pdf 
Scientific Journal of Nursing

25. Bernal-García M, Salamanca D, Pérez N, Quemba M. Validez de contenido por juicio de expertos de un instrumento para medir percepciones físico-emocionales en la práctica de disección anatómica. Revista Educ Med. 2019; 2(20):2-8. https://doi.org/10.1016/j.edumed.2018.08.008

26. Juárez-Hernández LG, Tobón S. Análisis de los elementos implícitos en la validación de contenido de un instrumento de investigación. Revista Espacios. [Internet]. 2018 [Consultado el 29 de mayo del 2021]; 39(53):23-28. Disponible en: https://www.revistaespacios.com/cited2017/cited2017-23.pdf

27. García A, Antúnez A, Ibáñez SJ. Análisis del proceso formativo en jugadores expertos: validación de instrumento. Revista Internacional de Medicina y Ciencias de la Actividad Física y el Deporte. [Internet].2018 [Consultado el 28 de mayo del 2021]; 16(61):157-182. Disponible en: https://revistas.uam.es/rimcafd/article/ view/3782/4072

28. García-Guadalupe JJ. 1 primer congreso nacional de educación, “educación para el éxito siglo XXI”. Revisión de la instrumentación de las tesis de la escuela de posgrado de la universidad nacional de educación durante el periodo 2012-201. [Internet]. Quito: UNAE; 2018 [Consultando el 29 de mayo del 2021]p. 6. Disponible en: https://unae.edu.ec/investigacion/quienes-somos/

29. Collet C, Nascimento JV, Folle A, Ibáñez SJ. Construcción y validación de un instrumento para el análisis de la formación deportiva en voleibol. Cuadernos de Psicología del Deporte. [Internet]. 2019 [Consultado el 29 de mayo del 2021]; 19(1):178-191. disponible en: https://revistas.um.es/cpd/article/view/326361

30. Práxedes A, Sevil J, Moreno A, Del villar F, García-González L. Niveles de actividad Física en estudiantes universitarios: diferencias en función del género, la edad y los estados de cambio. Revista iberoamericana de psicología del Ejercicio y el Deporte. [Internet]. 2016 [consultado 24 de mayo 2021];11(1):123-132. Disponible en: https://dialnet.unirioja.es/servlet/articulo?codigo $=5287993$

31. Molina-Arellano CX, Andrade-Salas HM. El ejercicio para jóvenes universitarios. Rev. UNIMAR [Internet]. 2017 [consultado 26 de mayo de 2021]; 34(1). Disponible en: http://editorial.umariana.edu.co/revistas/in $\neg-$ dex.php/unimar/article/view/1142

32. Dominguez-Lara SA, Bonifacio-Vilela M, Caro-Salazar A. Prevalencia de ansiedad ante exámenes en una muestra de estudiantes universitarios de lima metropolitana. Psicología [Internet]. 2019 [Consultado el 29 de mayo de 2021]; 6(2):45-56. Disponible en: https://revistas.ucsp.edu.pe/index.php/psicologia/article/view/155

33. Reyes, P. Estrategia de promoción del autocuidado de la salud en estudiantes de Medicina: Universidad Surcolombiana 2010-2011. [Internet]. La Habana, Cuba. Escuela Nacional de Salud Pública, 2016 [Consultado el 30 de mayo del 2020]. Disponible en: https://www.usco.edu.co/archivosUsuarios/19/publicacion/facultad de salud/acuerdo/acuerdo_021 de 2019.pdf 